Melissa POLLOCK, The Lion, the Lily, and the Leopard: The Crown and Nobility of Scotland, France, and England and the Struggle for Power (1100-1204)

\title{
Amélie Rigollet
}

\section{OpenEdition}

\section{Journals}

Édition électronique

URL : https://journals.openedition.org/ccm/5414

DOI : $10.4000 / \mathrm{ccm} .5414$

ISSN : 2119-1026

\section{Éditeur}

Centre d'études supérieures de civilisation médiévale/Université de Poitiers

\section{Édition imprimée}

Date de publication : 1 décembre 2020

Pagination : 299-300

ISBN : 978-2-490783-07-6

ISSN : 0007-9731

\section{Référence électronique}

Amélie Rigollet, "Melissa polıock, The Lion, the Lily, and the Leopard: The Crown and Nobility of Scotland, France, and England and the Struggle for Power (1700-1204) ", Cahiers de civilisation médiévale [En ligne], 252 | 2020, mis en ligne le 02 décembre 2020, consulté le 25 novembre 2022. URL : http:// journals.openedition.org/ccm/5414; DOI : https://doi.org/10.4000/ccm.5414

\section{(c)}

Creative Commons - Attribution - Pas d'Utilisation Commerciale - Pas de Modification 4.0 International - CC BY-NC-ND 4.0

https://creativecommons.org/licenses/by-nc-nd/4.0/ 
Melissa Pollock, The Lion, the Lily, and the Leopard: The Crown and Nobility of Scotland, France, and England and the Struggle for Power (1100-1204), Turnhout, Brepols (Medieval Identities: Socio-Cultural Spaces, 4), 2015.

Melissa Pollock analyse les relations royales et aristocratiques qui lient l'Écosse à la France et l'Angleterre au XII $\mathrm{e}$., période où ces trois royaumes sont dirigés par une élite transfrontalière commune. L'analyse s'achève en 1204, lorsque l'Angleterre perd la Normandie, l'Anjou, la Touraine et le Maine. Cette année est considérée comme le moment de rupture brisant définitivement l'unité de cette élite gouvernante (p. 30).

L'a. débute son introduction (p. 1-31) en remettant en question la définition de l'Auld Alliance - la Vieille Alliance renouvelée régulièrement entre la France et l'Écosse entre la fin du XIII ${ }^{\mathrm{e}} \mathrm{s}$. et le XVI ${ }^{\mathrm{e}} \mathrm{s}$. - en tant que réaction tardive de l'Écosse et de la France face aux agressions de l'Angleterre : les deux royaumes ont des liens anciens, formés dès 1066 (p. 1). L'a. s'intéresse aux stratégies d'adaptabilité et de mobilité mises en place par les familles aristocratiques pour préserver leurs intérêts territoriaux par-delà les frontières des royaumes au cours du XII ${ }^{\mathrm{e}}$ s. (p. 11). Son introduction s'achève par la présentation des sources utilisées, marquées par une nette différence de conservation entre les trois royaumes, caractérisées par la prépondérance des sources de la couronne anglaise. La rareté des sources administratives françaises - due au manque de centralisation et à la perte des bagages du roi Philippe poursuivi par Richard en 1194 (p. 15-16) - et écossaises - limitées aux chartes royales en l'absence d'une administration centralisée (p. 16-19) - est contrebalancée par l'importance des sources ecclésiastiques, mais aussi par les mouvements littéraires de la fin du XII ${ }^{\mathrm{e}}$ s. (p. 23), dont les emprunts croisés sont fréquents (p. 25).

La structure de l'ouvrage suit un plan thématicochronologique divisé en huit chapitres, explorant les questions matrimoniales et identitaires selon le découpage temporel imposé par les événements politiques et les règnes du XII ${ }^{\mathrm{e}} \mathrm{s}$., notamment lorsque les tensions entre les trois royaumes implosent, telles la guerre de succession de 1135-1153 (chap. 3), la guerre de 1173 (chap. 5) et la perte de la Normandie en 1204 (chap. 8). Le premier chapitre positionne le cadre conceptuel en considérant les notions d'image et d'identité (p. 33-53). L'a. justifie son utilisation du terme d'anglo-français au détriment de celui d'anglonormand par une conception géographique élargie (p. 34-35), puis elle questionne la notion d'ethnicité anglo-française et anglaise (p. 36-39) en récapitulant les conclusions historiographiques actuelles (p. 35 et p. 42).

Les stratégies matrimoniales constituent un fil directeur majeur de l'ouvrage, reposant sur de nombreuses études de cas. Le chap. 2 présente, à travers des exemples abondants, les relations matrimoniales écossaises dans la première moitié du XII ${ }^{\mathrm{e}} \mathrm{s}$. (p. 55-110) en étudiant le rôle joué par le roi Henri I ${ }^{\text {er }}$ d'Angleterre (1100-1135), surnommé le «marieur» (angl. matchmaker), qui utilise les unions pour maintenir les liens d'amitié (p. 64).

L'a. examine les relations d'interdépendance entre les trois pouvoirs royaux et l'aristocratie transmanche (angl. Cross-Channel), rythmées par les transformations imposées par les alliances ou les conflits. Le chap. 3 (p. 111-147) montre les fractures relationnelles entre l'Écosse, l'Angleterre, et la France pendant la guerre de succession, de 1135 à 1153 . Le roi David Ir d'Écosse (1124-1153), pendant la "révolution davidienne» (en angl. Davidian Revolution) (Geoffrey Wallis Stewart BARRow, « David I of Scotland: The Balance of New and Old », Scotland and Its Neighbours in the Middle Ages, Londres/Rio Grande Ohio, Hambledon Press, 1992, p. 45-65; Michael LyNCH, Scotland: A New History, Londres, Century, 1991 ; Robert BARTLETT, TheMaking of Europe: Conquest, Colonization and Cultural Change, 950-1350, Londres, Penguin, 1993; Robert Ian Moore, The First European Revolution, c. 970-1215, Oxford/Malden, Blackwell (The Making of Europe), 2000; Geoffrey Wallis Stewart BARRow, The Kingdom of the Scots: Government, Church and Society from the Eleventh to the Fourteenth Century, Édimbourg, Édinburgh University Press, 2003; Richard Oram, David: The King Who Made Scotland, Stroud, Tempus (Tempus Scottish Monarchs), 2004.) organise l' «anglo-normandisation» de sa cour et choisit de «coloniser» (p. 147) l'Écosse par l'implantation de familles anglo-françaises, unions qui rendirent par la suite l'alliance franco-écossaise «si personnelle» (p. 147). Le chap. 4 (p. 149-198) explore les conséquences de l'arrivée au pouvoir du roi Henri II d'Angleterre (1154-1189), le nouvel équilibre entre les trois royaumes provoquant des redécoupages territoriaux qui aboutissent à des arrangements matrimoniaux. Les exemples fournis illustrent les positionnements de l'aristocratie, soit individuel, tel le cas de Philippe de Coulommiers 
(p. 155), soit prosopographique, telles les alliances bretonnes (p. 178-196).

Le chap. 5 (p. 199-285) observe l'implication de l'Écosse dans la guerre de 1173-1174, dont la participation auprès de la noblesse anglo-française s'intensifie (p. 201). L'a. développe, parmi de nombreux exemples, le parcours de la famille de Montbray (anc. fr. Moubray) pour illustrer l'atout que représentent les réseaux lignagers et territoriaux étendus (p. 202-210). Le chap. 6 (p. 287-336) étudie la période suivant la rébellion de 1173-1174, marquée par l'absence de contact entre l'Écosse et la France pendant 25 ans. L'a. considère le positionnement du roi Guillaume I ${ }^{\text {er }}$ d'Écosse (1165-1214), jusqu'à la mort du roi anglais Henri II en 1189, ainsi que l'influence à la cour écossaise des familles «transmanches » (p. 287). Les relations stables entre le roi Guillaume I ${ }^{\text {er }}$ d'Écosse et le roi Richard d'Angleterre (1189-1199), au détriment de liens français, font 1'objet du chap. 7 (p. 337-381). L'arrivée au pouvoir du roi Jean d'Angleterre (1199-1216) en 1199 entraine l'étiolement des relations anglo-écossaises, le roi Guillaume renouant alors le dialogue avec la France (p. 381). Le dernier chapitre, le huitième, analyse l'impact de la perte de la Normandie sur les interconnexions entre les trois royaumes (p. 383-413). La détérioration des relations anglo-écossaises (p. 388) est tempérée par le positionnement du frère du roi Guillaume, le comte David de Huntingdon (†1219), devenu un «homme du roi Jean» (p. 409) dont la loyauté est récompensée. Parallèlement, les liens franco-écossais sont distendus, comme en témoigne le projet infructueux d'union entre Alexandre, fils du roi Guillaume d'Écosse, et Marie de France, fille de Philippe Auguste (p. 392). L'isolement de l'Écosse coïncide avec celui de l'Angleterre, provoqué par la dégradation des liens entre le roi Jean et le Continent, suite à l'affaire provoquée par son mariage avec Isabelle d'Angoulême en 1200 (p. 389 et p. 395).

L'a. résume en conclusion (p. 415-419) l'idée centrale de son ouvrage. Après 1204, survient un changement profond, «l'aristocratie d'Écosse, d'Angleterre et de France évolue, de groupes familiaux partageant des coutumes et une langue commune à des régimes plus indépendants, séparés les uns des autres» (p. 416). Selon l'a., la formation d'une identité «nationale» dans chacun de ces trois royaumes aurait été ralentie par la permanence au pouvoir d'une élite aristocratique transfrontalière. Avant 1204, ces sentiments unitaires étaient dilués, du fait de la structuration transmanche des seigneuries et des lignages (p. 417).

L'ouvrage est complété par vingt tables de filiation (liste p. VII), réparties au fil de l'ouvrage (fig. 1 à 17) ou placées en annexe (fig. 18 à 20, p. 421-423). Quatre cartes localisant des propriétés croisées de plusieurs seigneurs ponctuent le texte. La bibliographie (p. 425-492) distingue les sources archivistiques (p. 425-426), imprimées (p. 427-446) et secondaires (p. 446-492). L'index (p. 493-523) précise les relations entre les individus, le positionnement généalogique et les hypothèses d'identification. Tous les individus indiqués dans l'ouvrage ne sont pas inclus, seuls les noms ayant plus d'une occurrence sont listés. L'a. renvoie utilement à un index complet de tous les individus et toponymes mentionnés dans son ouvrage, ce répertoire étant disponible en ligne (https://st-andrews.academia.edu/melissapollock).

Amélie Rigollet UMR 7302 - CESCM Université de Poitiers 